\title{
Anatomy of the Lymphatic System and the Lymphosome Concept with Reference to Lymphedema
}

\author{
Hiroo Suami, MD, $\mathrm{PhD}^{1}$ Mario F. Scaglioni, $\mathrm{MD}^{2}$ \\ ${ }^{1}$ Australian Lymphoedema Education Research Treatment Centre \\ (ALERT), Faculty of Medicine and Health Sciences, Macquarie \\ University, Sydney, NSW, Australia \\ 2 Department of Plastic and Hand Surgery, University Hospital Zurich, \\ Zurich, Switzerland
}

Semin Plast Surg 2018;32:5-11.

\begin{abstract}
Address for correspondence Hiroo Suami, MD, PhD, Australian Lymphoedema Education Research Treatment Centre (ALERT), Faculty of Medicine and Health Sciences, Level 1, 75 Talavera Road, Macquarie University, NSW 2109, Australia (e-mail: hiroo.suami@mq.edu.au).
\end{abstract}

\begin{abstract}
Keywords

- lymph capillary

- precollector

- lymph collecting vessel

- lymphosome

- sentinel node

Precise knowledge of the lymphatic system normal anatomy is essential for understanding what structural changes occur in patients with lymphedema. In this article, the authors first review previous anatomical studies and summarize the general anatomy of the lymphatic system and lymphatic pathways in the upper and lower extremities. Second, they introduce their new anatomical concept, the "lymphosome," which describes how the lymphatic vessels in a particular region connect to the same subgroup of regional lymph nodes. In addition, they describe the anatomical relationship between the perforating lymphatic vessels and arteries. In the last section, they explain the anatomical changes in the lymphatics after lymph node dissection, with reference to secondary lymphedema.
\end{abstract}

The lymphatic system is distributed throughout the entire human body, except for the bone marrow, cartilage, and the central nervous system (not including the dura mater). The lymphatic capillary network wraps around the surface of the body and also lines the internal surface of the gastrointestinal and respiratory tract. The major role of the lymphatic system is to return protein deposits and extra tissue fluid extravasated from the blood capillaries to the interstitial tissues to feed into the blood circulation system to maintain fluid balance in the body. ${ }^{1}$ Another important function of the lymphatic system is to convey germs and pathogens to the lymph nodes via the lymphatic vessels to activate the immunological chain reaction that defends the body.

Our current knowledge of the lymphatic system anatomy relies largely on the results of cadaver studies by Sappey, published in $1874 .^{2}$ In his studies, mercury was used to delineate the lymphatic system, but the toxicity of mercury meant that similar studies were never attempted, and his findings have never been re-evaluated. While Sappey's atlases are still an excellent resource for understanding general lymphatic drainage in different parts of the body, recent clinical data obtained using lymphoscintigraphy sometimes contradict the conventionally acknowledged lymphatic drainage pathways in the torso. ${ }^{3}$

Knowledge of the normal anatomy of the lymphatic system is crucial for predicting which lymph nodes may be the site of metastatic disease after a primary tumor and for understanding the pathological changes that occur in lympedema. Lymphedema is a disease regarded as a malfunction of the lymphatic system that presents as swelling in body regions, particularly the limbs. The causes of lymphedema are divided into congenital (primary lymphedema) and acquired factors (secondary lymphedema). Congenital lymphedema represents various structural abnormalities: aplasia and hyperplasia associated with malfunctioning valves in the lymphatic vessel. Gene mutations associated with other vascular malformations are identified as mutations of FOXC2 or SOX18. ${ }^{4}$ Acquired factors include trauma, filariasis, and
Issue Theme Lymphedema Management; Guest Editors: Edward I. Chang, MD, FACS, Mark V. Schaverien, MBChB, MD, MSc, MEd, FRCS (Plast), and Jesse C. Selber, MD, MPH, FACS
Copyright $\odot 2018$ by Thieme Medical Publishers, Inc., 333 Seventh Avenue, New York, NY 10001, USA.

Tel: +1(212) 584-4662.
DOI https://doi.org/ 10.1055/s-0038-1635118. ISSN $1535-2188$. 
cancer treatment. Secondary lymphedema related to cancer treatment is triggered by breaking of the lymphatic vessels following lymph node dissection and/or radiation.

Decongestive therapy is the primary course of treatment for lymphedema. It includes manual lymphatic drainage (MLD), skin care, and wearing of compression garments. Alternatively, surgical treatment has been revisited recently, with the development of a super-microsurgical technique enabling lymphaticovenular anastomosis (LVA) and indocyanine green (ICG) fluorescence lymphography. A better understanding of the anatomy of the lymphatic system may lead to the development of more reliable surgical treatments.

\section{Structure of the Lymphatic System}

Lymphatic vessels are divided into three categories based on their structural characteristics: lymph capillaries, pre-collectors, and lymph-collecting vessels (-Fig. 1). ${ }^{5}$ The lymph capillaries (between 20 and $70 \mu \mathrm{m}$ in diameter) are located immediately beneath the epidermis. They do not have a valvular structure. The lymph capillary begins with a blind ending. The endothelial cells that form the lymph capillary connect with each other loosely in an overlapping pattern, like roof tiles. A fibrous structure called an anchoring filament connects the endothelial cell with the surrounding tissue. ${ }^{6}$ When the tissue increases in volume owing to extra interstitial fluid (edema), the anchoring filaments pull the endothelial cells outward so that the junctions between the cells open up to capture the extra interstitial fluid into the lumen.

The lymph capillaries connect to pre-collectors in the deeper layer of the dermis. Pre-collectors (between 70 and
$150 \mu \mathrm{m}$ in diameter) have a valvular structure that regulates the direction of lymph flow unidirectionally from the superficial to the deep layers. Pre-collectors converge within the dermis to form a larger vessel, exit beneath the dermis, and run vertically through the subcutaneous tissue. These vertical vessels are called efferent pre-collectors.

The pre-collectors connect to the lymph-collecting vessels, or collectors, in the subcutaneous fat layer. These collectors (between 150 and $500 \mu \mathrm{m}$ in diameter) run horizontally in the subcutaneous tissue and are larger than the lymph capillaries and pre-collectors. The lymph-collecting vessels have a three-layered wall made of endothelial cells, smooth muscle cells, and collagen fibers with fibroblasts that contracts rhythmically to propel lymph flow. The lymph-collecting vessels are subcategorized into superficial and deep vessels according to their anatomical relationship to the deep fascia. The deep lymph-collecting vessels accompany the arteries, whereas the superficial lymph-collecting vessels have no such preference. The superficial lymphcollecting vessels outnumber the deep lymph-collecting vessels, with the two types segregated from each other.

It is a common mistake to try to understand the lymphatic system by comparing it to the venous system. The venous system has a valvular structure, branching cutaneous veins and perforating veins that connect the superficial and deep veins. However, in the lymphatic system, the lymph-collecting vessels are more independent than veins, with fewer interconnections and branching at narrower angles. In addition, there is no bridge between the superficial and deep lymph vessels. The lymphatic system does have perforating vessels, but they do not join the superficial and deep lymph vessels. Also, veins increase in diameter in the proximal

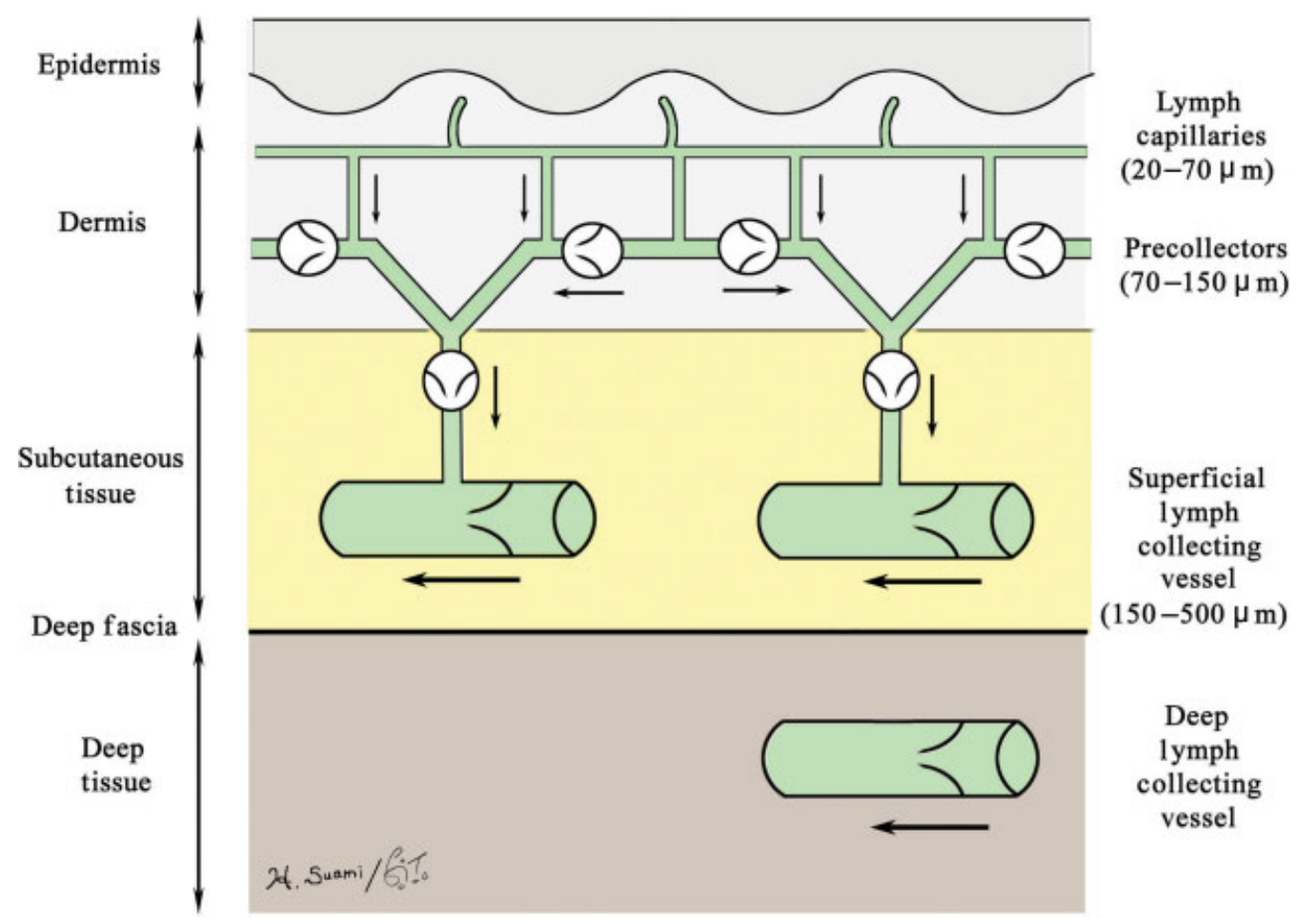

Fig. 1 Schematic diagram of the lymphatic system. (Adapted from Suami et al. ${ }^{5}$ Reproduced with permission.) 
region, but the lymphatic vessels are uniform in size between the distal and the proximal regions near the lymph nodes.

\section{Regional and Interval Lymph Nodes}

As a consequence of his anatomical studies with human cadavers, Bartels ${ }^{7}$ proposed the lymph node barrier theory that postulates that each lymphatic vessel connects to at least one lymph node before connecting to the vein. The lymph node is the organ that catches pathogenic organisms or cancer cells and provokes an immune response to combat them to protect the human body. The barrier theory explains how germs are released into the systemic blood circulation only after the lymph nodes have activated the body's immune response.

Lymph nodes are classified as 'regional' or 'interval', according to their location. Regional lymph nodes are groups of lymph nodes that form lymphatic basins into which lymph drains from different skin regions or organs. The regional lymph nodes are the target of lymph node dissection in cancer treatment to halt the spread of cancer cells, with neck dissection for tongue cancer, axillary dissection for breast cancer, and inguinal dissection for lower extremity melanoma. Interval lymph nodes are located in the limbs, and the lymph vessels pass through them on the way to the regional lymph nodes. The epitrochlear and popliteal nodes are wellknown examples, but there are several other interval lymph nodes in the deep lymphatic system located along the main arteries in the limbs.

In the regional lymph nodes, there are more afferent lymph-collecting vessels than efferent lymph-collecting vessels; however, in the interval lymph nodes, there is a similar number of each type. The functional difference between the regional and interval lymph nodes has been little studied, and the role of the interval lymph nodes in fighting malignant neoplasms is uncertain and still under debate.

\section{Superficial Lymphatic System in the Upper Extremities}

The superficial lymphatic system in the upper extremities originates in the lymph capillaries in the fingertips and palm. The lymph capillaries transition into pre-collectors in the dermis, with the efferent pre-collectors exiting the dermis and connecting to the superficial lymph-collecting vessels in the subcutaneous tissue. One to three superficial lymphcollecting vessels $(\sim 200 \mu \mathrm{m}$ in diameter) run along both sides of the fingers in the neurovascular bundle. All lymphcollecting vessels emerging from the fingers run axially in the dorsal hand in a layer slightly deeper than the cutaneous veins. The superficial lymph-collecting vessels are evenly spaced around the wrist joint and then gradually change course around the elbow toward the anteromedial aspect and run parallel to the basilic vein in the upper arm (-Fig. 2)..$^{7}$ They connect to one or two dominant lymph nodes (sentry nodes) in the lateral axillary region (-Fig. 3). ${ }^{8}$ The axillary lymph node region comprises 20 to 40 regional lymph nodes.
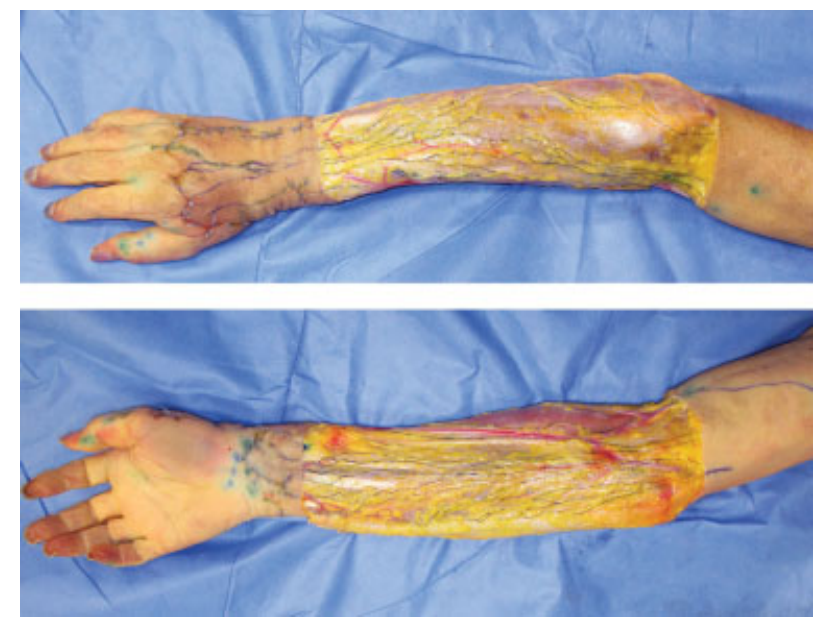

Fig. 2 Photos of an upper limb in a cadaver injected with dye into the collecting lymph vessels.

An alternative pathway of the superficial lymph collecting vessels skips the axillary lymph nodes and connects directly to the supraclavicular lymph nodes. One to two lymphatic vessels follow along the cephalic vein in the lateral upper arm and connect to the supraclavicular nodes after passing through the deltopectoral node, which is an interval lymph node located at the deltopectoral groove. This route approaches the subclavian vein from the cranial aspect. This area is outside the axillary node dissection range for breast cancer or melanoma treatment. It is postulated that

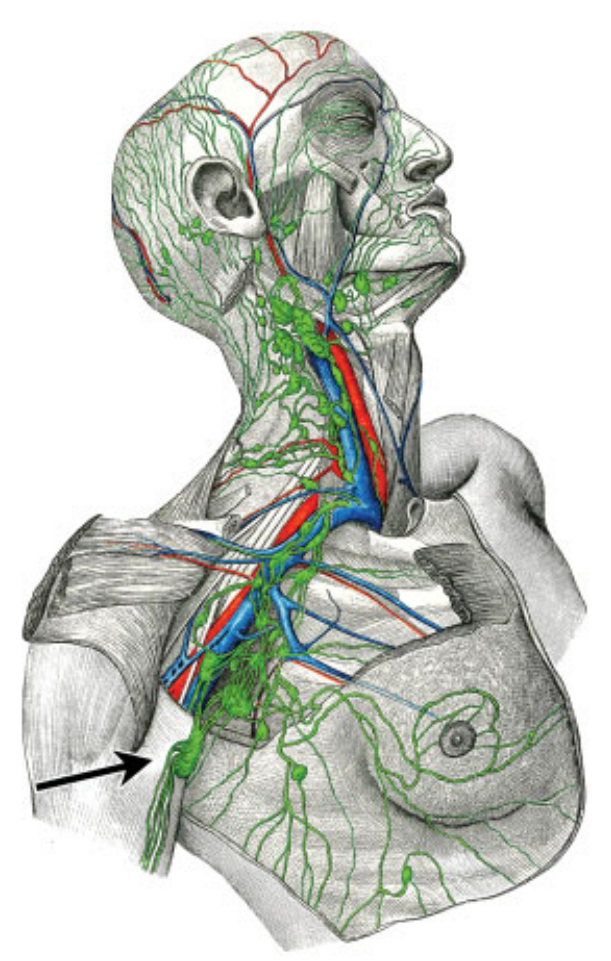

Fig. 3 Schematic diagram of the lymphatic system in the upper half of the body. An arrow points to the sentry lymph node of the upper extremity. (Color of the lymphatics added to the original diagram from Jossifow. ${ }^{8}$ ) 

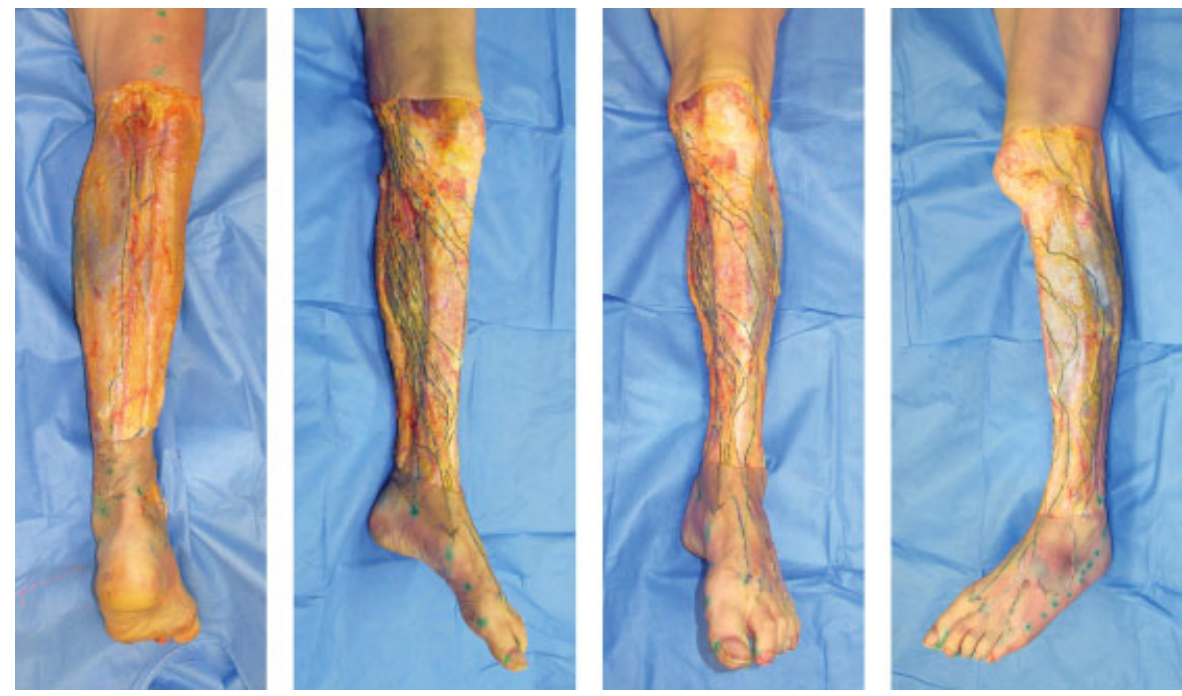

Fig. 4 Photos of a lower limb in a cadaver injected with dye into the collecting lymph vessels.

this supraclavicular pathway, if present, can work as an escape route to prevent upper-extremity lymphedema.

\section{Superficial Lymphatic System in the Lower Extremities}

Similar to the upper extremities, the superficial lymphatic system in the lower extremities originates in the lymph capillaries in the toes and soles of the feet. The diameter of the superficial lymph-collecting vessels in the foot is larger than that in the hand, between 300 and $700 \mu \mathrm{m}$. The vessels from the medial and anterior sides of the foot ascend axially, diverge, merge below the knee and then change course toward the anteromedial thigh, running parallel with the great saphenous vein above the knee (- Fig. 4). ${ }^{7}$ The vessels in the medial bundle connect to two or three dominant lymph nodes (sentry nodes) at the bottom of the femoral triangle, which is bordered by the lateral border of the adductor longus, the medial border of the sartorius, and the inguinal ligament ( $\mathbf{- F i g . 5})^{8}$

The lymph capillaries in the lateral heel region converge to become one to three superficial lymph-collecting vessels that run along the small saphenous vein in the calf region. These vessels are separate from those in the medial bundle and connect to the popliteal lymph node, which is an interval lymph node located in the deep layer of the knee fossa. Efferent lymphatic vessels of the popliteal node ascend along the deep femoral artery and bypass the sentry lymph nodes in the superficial inguinal region.

\section{Lymphosome Concept}

Historically, anatomical studies of the lymphatic system flourished with the mercury injection technique from the $17^{\text {th }}$ to $20^{\text {th }}$ centuries, but mercury's toxicity eventually brought an end to this practice. We developed a novel radiological method for demonstrating the lymphatic system in cadaveric specimens by using hydrogen peroxide, radiocontrast medium, and microinjection technique. ${ }^{9,10}$ Using this method, we found substantial anatomical differences between the lymphatic and blood systems.

Each superficial lymph-collecting vessel runs in a straight path toward its corresponding lymph node. These vessels sometimes diverge and re-converge, demonstrating their interconnections; however, the interconnections are much fewer than those found in blood vessels. No lymph vessels

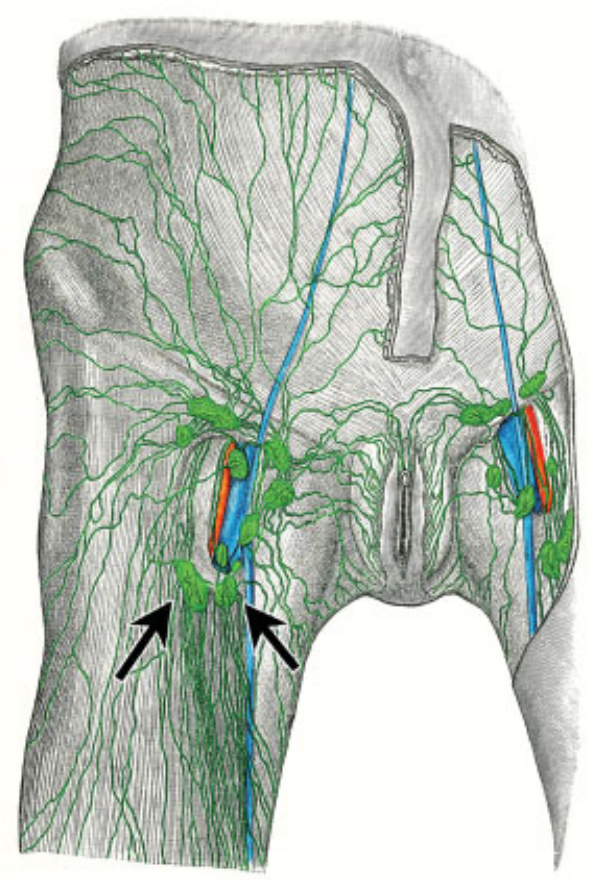

Fig. 5 Schematic diagram of the lymphatic system in the inguinal region. Arrows point to the sentry lymph nodes of the lower extremity. (Color of the lymphatics added to the original diagram from jossifow. ${ }^{8}$ ) 
connect the superficial and deep lymph-collecting vessels. The superficial lymph-collecting vessels do not overlap, but are arranged in a plane. These characteristics enable us to divide the skin into territories that correlate with their lymph basins.

The following steps were taken to define the superficial lymphatic territories (lymphosomes) in a cadaver model. ${ }^{11}$ First, each superficial lymph-collecting vessel was identified in the peripheral region using hydrogen peroxide with or without dye. Radiopaque material was injected directly into the vessel using the microinjection technique. The course of each lymphatic vessel was followed by dissecting the vessel and following it through to its corresponding lymph node (sentinel node). If several lymph nodes were found in one lymph basin in the inguinal or axillary region, those lymph nodes were divided into subgroups according to their anatomical location in the upstream region. Each group of lymph nodes was color-coded, and the corresponding lymph vessels were coded in the same color by working backward from the node. Thereby the skin was divided into superficial lymphatic territories (lymphosomes) (-Fig. 6).

Although minor differences in lymphatic anatomy between individuals and sides of the body have been consistently acknowledged, we succeeded in defining lymphosomes by dividing the regional nodes into subgroups. We expect the lymphosome concept to increase understanding of the lymphatic system, to provide a template to more accurately interpret lymphoscintigraphy, and to define suitable lymph node donor sites for vascularized lymph node transfer.

\section{Perforating Lymphatic Vessels}

There are no lymphatic vessels directly connecting the superficial and deep lymph-collecting vessels under normal conditions. However, there is another type of lymph vessel that runs in the subcutaneous tissue and joins with the deep lymph-collecting vessels, but does not form a connection between superficial and deep vessels. In our study, we named these vessels "perforating lymph vessels" because they run along the perforating artery and perforate the deep fascia (-Fig. 7)..$^{5}$ We discovered these perforating lymph vessels when we dissected breast specimens and looked for the lymph vessels that drain from the breast to the internal mammary lymph nodes. In our specimens, the perforating lymph vessels ran along cutaneous branches of the internal mammary artery, which is the vascular pedicle of the deltopectoral flap. Although perforating lymph vessels in the torso have often been identified by lymphoscintigraphy, the corresponding lymph nodes were usually described as
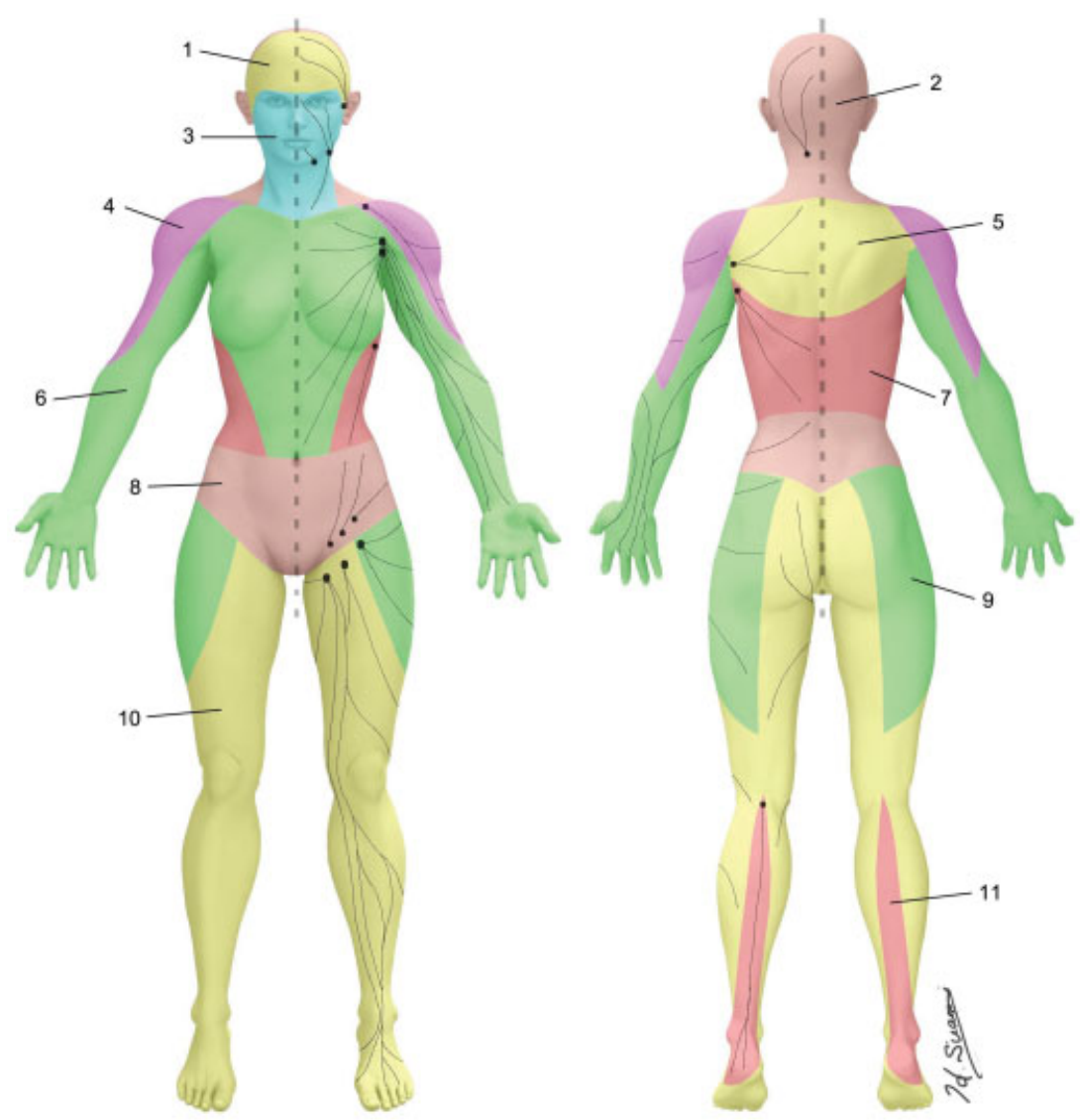

Fig. 6 Lymphosomes of the body. The lymphatic territories are demarcated according to their corresponding lymphatic basins: 1. temporal, 2. occipital, 3. submental, 4. subclavicular, 5. subscapular, 6. lateral axillary, 7. pectoral, 8. superior inguinal, 9. lateral inguinal, 10. inferior inguinal, 11. popliteal. (Reproduced with permission of Hiroo Suami, 2018.) 


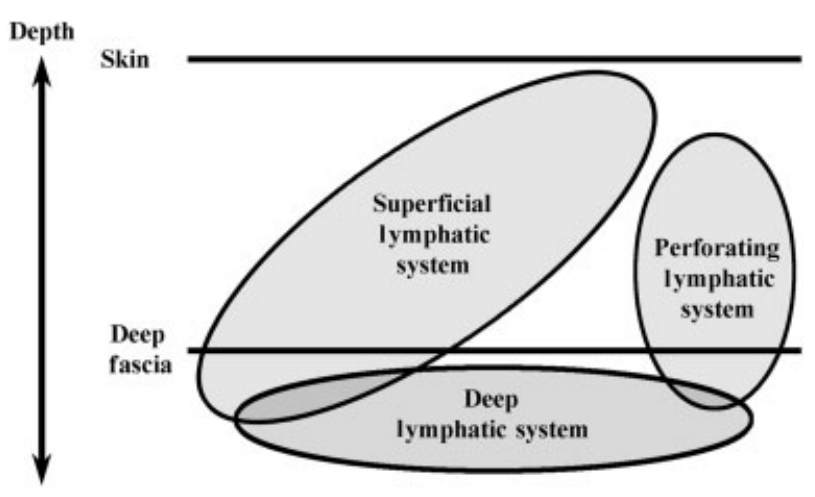

Fig. 7 Our representation of the three types of lymphatic drainage: superficial, deep, and perforating lymphatic systems. (Adapted from Suami et al. ${ }^{5}$ Reproduced with permission.)

"unexpected lymph nodes," because they were located in neither the axillary nor inguinal regions. These unexpected lymph nodes were found in the vertebral, internal mammary, and triangular muscular space nodes following injection of a radiotracer in the lumbar back region, the upper abdominal region, and the upper back region, respectively. ${ }^{12}$ In these cases, the unexpected lymph drainage was thought to pass through the perforating lymph vessels running along the cutaneous branches of the lumbar artery, the superficial superior epigastric artery and the circumflex scapular artery, respectively. A better understanding of the perforating lymph vessels would enable more accurate prediction of skin cancer metastases.

\section{Postoperative Changes in Lymph Structure}

The structure of the lymphatic system changes significantly after lymph node dissection, even though patients may not display any clinical symptoms. Severed lymph vessels regenerate from a stump and attempt to recanalize. ${ }^{13,14}$ A specific indicator of lymphedema is "dermal backflow," which can be detected by an intradermal injection of dye, radiotracer (lymphoscintigraphy), and ICG (fluorescent lymphography). When the superficial lymph-collecting vessels are obstructed in the proximal region following oncologic treatment, changes in the lymphatic system also occur in the distal regions, including the hands or feet in severe cases. In lymphedema, lymph fluid flows backward from the superficial lymph-collecting vessel to the pre-collectors and lymph capillaries in the skin via the efferent pre-collectors. Valve insufficiency in the superficial lymph-collecting vessels and pre-collectors causes the reflux of lymph fluid into the skin. The lymph capillaries and pre-collectors in the skin are dilated, and in the advanced stages of lymphedema, the superficial lymph-collecting vessels deteriorate and disappear. Notably, dermal backflow can be seen not only in lymphedematous limbs, but also in the subclinical phase in secondary lymphedema.

In mild cases of lymphedema, areas of dermal backflow are localized because some lymph-collecting vessels are still patent, so the dermal backflow can work as a detour route to bridge obstructed and intact lymph-collecting vessels. In advanced cases, there are fewer superficial lymph-collecting vessels, and the lymph fluid is carried by dermal backflow. The degree of lymphedema severity correlates with the amount of damaged lymph-collecting vessels (- Fig. 8). ${ }^{15}$

\section{Conclusion}

Due to the technical difficulties of visualizing the lymphatic system in cadaver dissection studies, anatomical study of the lymphatic system has been held back for over 100 years. However, the authors' use of the radiographic cadaver microinjection technique and ICG fluorescence lymphography in the clinical setting can now provide further detail about the lymphatics. This article provides a brief overview of the anatomy of the lymphatic system. The lymphosome concept and new knowledge about the perforating lymphatic vessels can be a useful tool in accurately predicting the location of the sentinel nodes in skin or breast cancer patients and in promoting understanding of the pathophysiology of lymphedema.

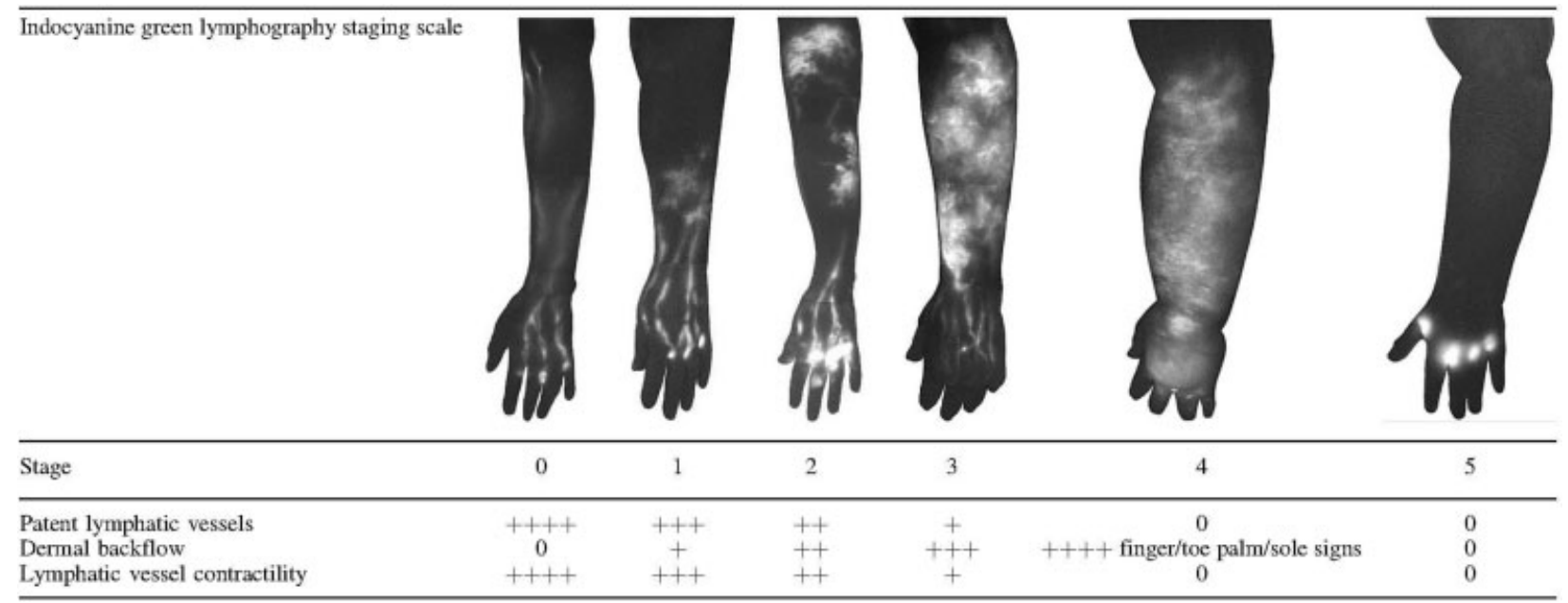

Fig. 8 ICG lymphography staging scale. (Adapted from Nguyen et al. ${ }^{5}$ Reproduced with permission.). ICG, indocyanine green. 


\section{Conflict of Interest}

None.

\section{References}

1 Yoffey JM, Courtice FC. Lymphatics, Lymph and the Lymphomyeloid Complex. London and New York: Academic Press; 1970

2 Sappey MPC. Anatomie, Physiologie, Pathologie Des Vaisseaux Lymphatiques. Paris: AdrienDelahaye; 1874

3 Uren RF, Hoefnagal CA. Lymphoscintigraphy. In: Thompson JF, Morton DL, Kroon BBR, eds. Textbook of Melanoma. London and New York: Taylor \& Francis; 2004

4 Rockson SG. Genetic prospects for lymphedema management. In: Lee BB, Bergan J, Rockson SG, eds. Lymphedema A Concise Compendium of Theory and Practice. London: Springer; 2011

5 Suami H, Pan WR, Mann GB, Taylor GI. The lymphatic anatomy of the breast and its implications for sentinel lymph node biopsy: a human cadaver study. Ann Surg Oncol 2008;15(03): 863-871

6 Leak LV. Electron microscopic observations on lymphatic capillaries and the structural components of the connective tissuelymph interface. Microvasc Res 1970;2(04):361-391

7 Bartels P. Das Lymphgefasssystem. Jene: Gustav Fischer; 1909
8 Jossifow GM. Das Lymphgefäßsystem des Menchen. Jena: Verlag von Gustav Fischer; 1930

9 Suami H, Taylor GI, Pan WR. A new radiographic cadaver injection technique for investigating the lymphatic system. Plast Reconstr Surg 2005;115(07):2007-2013

10 Suami H, Taylor GI, O'Neill J, Pan WR. Refinements of the radiographic cadaver injection technique for investigating minute lymphatic vessels. Plast Reconstr Surg 2007;120(01):61-67

11 Suami H. Lymphosome concept: anatomical study of the lymphatic system. J Surg Oncol 2017;115(01):13-17

12 Uren RF, Thompson JF, Howman-Glies RB. Lymphatic Drainage of the Skin and Breast. Amsterdam: Harwood Academic Publishers; 1999

13 Gray JH. Studies of the regeneration of lymphatic vessels. J Anat 1940-40;74(Pt 3):309-335

14 Suami H, Scaglioni MF, Dixon KA, Tailor RC. Interaction between vascularized lymph node transfer and recipient lymphatics after lymph node dissection-a pilot study in a canine model. J Surg Res 2016;204(02):418-427

15 Nguyen AT, Suami H, Hanasono MM, Womack VA, Wong FC, Chang EI. Long-term outcomes of the minimally invasive free vascularized omental lymphatic flap for the treatment of lymphedema. J Surg Oncol 2017;115(01):84-89 